\title{
SISTEMAS DE MANEJO E QUALIDADE ESTRUTURAL DE LATOSSOLO ROXO¹
}

\author{
MARX LEANDRO NAVES SILVA ${ }^{2}$, NILTON CURI ${ }^{2}$ e PHILIPPE BLANCANEAUX ${ }^{3}$
}

\begin{abstract}
RESUMO - Objetivou-se, com este estudo, avaliar a qualidade estrutural de um Latossolo Roxo, pelos seguintes atributos: distribuição do tamanho dos agregados, diâmetro médio geométrico dos agregados, resistência dos agregados ao impacto das gotas de chuva simulada, e resistência à penetração, em sistemas de plantio direto e cultivo convencional, tendo como testemunha a floresta nativa. Entre os métodos utilizados, a energia cinética necessária para destruir os agregados foi a que proporcionou maior discriminação entre os sistemas de manejo estudados. Na camada superficial, o sistema plantio direto propiciou agregados maiores e mais resistentes, e os valores do diâmetro médio geométrico e a energia cinética necessária para destruir agregados foram cerca de 1,37 e 2,4 vezes maiores do que no cultivo convencional, respectivamente. Valores críticos de resistência à penetração foram observados no que diz respeito ao cultivo convencional e ao plantio direto, na camada de $5-20 \mathrm{~cm}$ de profundidade. Por outro lado, na camada de $0-5 \mathrm{~cm}$, o plantio direto apresentou valor baixo de resistência à penetração, o que está relacionado com seu teor elevado de matéria orgânica e com material vegetal em decomposição na superfície do solo. Estes aspectos ressaltam o efeito benéfico deste sistema, pois contribuem para o manejo sustentado do solo.
\end{abstract}

Termos para indexação: estrutura do solo, porosidade do solo, permeabilidade, características físicoquímicas do solo, cultivo, plantio direto.

\section{MANAGEMENT SYSTEMS AND STRUCTURAL QUALITY OF A DUSKY-RED LATOSOL (OXISOL)}

\begin{abstract}
This study aimed to evaluate the structural quality of a Dusky-Red Latosol (Oxisol) by the following attributes: aggregate-size distribution, geometrical average diameter of aggregates, aggregates resistance to simulated raindrops impact, and penetration resistance, under no-till and conventional till systems, using the native forest as reference trial. Among the utilized methods, the kinetic energy necessary for destructing the aggregates presented the best distinction among the studied management systems. In the superficial layer, the no-till revealed bigger and more resistant aggregates, being its values of geometrical average diameter and kinetic energy necessary for destructing the aggregates around 1.37 and 2.4 times higher than the conventional till. Critical values for penetration resistance were observed for conventional till and no-till systems at 5-20 cm depth. On the other hand, in the $0-5 \mathrm{~cm}$ layer, the no-till presented a low value for penetration resistance, in accordance with its elevated amount of organic matter, associated with vegetal material in decomposition at soil surface. These aspects emphasize the beneficial effect of this system for the structural quality, contributing to the sustainable management of the soil.
\end{abstract}

Index terms: soil structure, soil porosity, permeability, soil physical-chemistry characteristics, cultivation, direct sowing.

\footnotetext{
${ }^{1}$ Aceito para publicação em 5 de abril de 2000. Trabalho apresentado no XXVI Congresso Brasileiro de Ciência do Solo, Rio de Janeiro, RJ, 20 a 26 de julho de 1997.

${ }^{2}$ Eng. Agrôn., D.Sc., Dep. de Ciência do Solo, Universidade Federal de Lavras (UFLA), Caixa Postal 37, CEP 37200-000 Lavras, MG. Bolsista do CNPq. E-mail: marx@ufla.br, niltcuri@ufla.br

${ }^{3}$ Geólogo, D.Sc., Institut de Recherche pour le Development (IRD), Rue La Fayette, 213, Cedex 10, 75480, Paris, France. E-mail: blancane@bondy.ird.fr
}

\section{INTRODUÇ̃̃O}

A adoção de sistemas conservacionistas de manejo do solo, como o plantio direto, tem sido apresentada como uma opção para assegurar a sustentabilidade do uso agrícola dos Latossolos no Brasil. Entretanto, é freqüente o uso de sistema de cultivo denominado convencional, que, basicamente, consiste no uso excessivo de arados e grades no preparo 
do solo, especialmente a grade aradora, que diminui a estabilidade dos agregados causando sua destruição (Machado et al., 1981; Reinert et al., 1984). $\mathrm{O}$ uso de metodologias que quantifiquem e qualifiquem as condições estruturais do solo nos vários sistemas de manejo é importante na avaliação da qualidade do solo, considerada um indicador da sustentabilidade dos sistemas de uso e manejo (Arshad et al., 1996; Harris et al., 1996).

Os atributos químicos e físicos exercem influência sobre a agregação e a estabilidade estrutural do solo. Silva \& Mielniczuk (1998) avaliaram os efeitos de sistemas de cultivo e dos atributos de um Latossolo Roxo e um Podzólico Vermelho-Escuro, ambos com textura muito argilosa, na estabilidade de agregados. Eles concluíram que apesar de os teores de carbono orgânico, $\mathrm{Fe}$ e $\mathrm{Al}$, argila e grau de dispersão das argilas afetarem a agregação do solo, foram insuficientes para explicar as variações no diâmetro médio geométrico sob os diferentes sistemas de cultivo.

A reestruturação do solo depende do sistema de manejo que será usado no preparo do mesmo. A adoção de sistemas de manejo que mantenham a proteção do solo através do contínuo aporte de resíduos orgânicos é fundamental para a manutenção de uma boa estrutura. $O$ contínuo fornecimento de material orgânico serve como fonte de energia para a atividade microbiana, que atua como agente de estabilização dos agregados (Campos et al., 1995). Castro Filho et al. (1998), estudando um Latossolo Roxo de Londrina, no Paraná, concluíram que o acúmulo de resíduos vegetais na superfície como conseqüência da adoção do sistema de plantio direto melhorou o estado de agregação graças ao incremento do teor de carbono orgânico, sobretudo na camada de 0-10 cm, independentemente da sucessão de culturas. Os valores de diâmetro médio geométrico dos agregados foram significativamente superiores em relação aos do plantio convencional.

Carpenedo \& Mielniczuk (1990), estudando a estabilidade estrutural dos agregados em condições de mata e campo nativo, verificaram que houve redução da agregação quando os solos foram submetidos à lavração e à gradagem para o cultivo de trigo e soja. Segundo Da Ros et al. (1997), após cinco anos de cultivo, o diâmetro médio geométrico dos agre- gados no tratamento com plantio direto foi estatisticamente equivalente ao do campo nativo, diminuindo com o aumento da intensidade de preparo do solo, com valores de 2,96 vezes menores no preparo convencional comparado ao campo nativo. Campos et al. (1995) constataram que no sistema de plantio direto o diâmetro médio geométrico dos agregados foi cerca de duas vezes maior que no sistema de plantio convencional.

A agregação do solo avaliada por meio da estabilidade dos agregados em água e resistência ao impacto das gotas de chuva proporciona melhor correlação com a erodibilidade do solo, seguida da porcentagem de agregados $>1 \mathrm{~mm}$. Isso acontece porque, quanto maior for o agregado, maiores serão o diâmetro médio geométrico e os espaços porosos entre eles, aumentando a infiltração e diminuindo a erosão (Angulo et al., 1984). Alvarenga et al. (1986) chegaram à conclusão semelhante, ao usar a porcentagem de agregados ( $>2 \mathrm{~mm}$ ) para expressar a agregação do solo. Harris et al. (1996) utilizaram a porcentagem de agregados estáveis ( $>2 \mathrm{~mm}$ ) em água para avaliar a qualidade do solo em sistemas de manejo; segundo esses autores, os valores foram 40, 26 e $19 \%$, para sistema conservacionista, plantio direto e solo arado, respectivamente. Segundo Silva et al. (1998), o método do impacto de gotas de chuva simulada apresenta bom desempenho e proporciona maior discriminação entre os tratamentos em relação ao método de estabilidade de agregados. Segundo esses autores, o cerrado nativo apresenta agregados maiores e mais estáveis, sendo necessária maior energia cinética para destruí-los em comparação aos sistemas de rotação milho-adubo verde, onde a gramínea mostrou maior ação agregante que as leguminosas (Silva \& Mielniczuk, 1998).

A resistência do solo à penetração aumenta com a compactação, e é restritiva ao crescimento radicular acima de certos valores que variam de 1,5 a 3,0 Mpa (Grant \& Lanfond, 1993) e de 2,0 a 4,0 MPa (Arshad et al., 1996), sendo ponderados valores superiores em plantio direto, devido à maior continuidade de poros e homogeneidade do solo. Valores entre 2,0 e 3,5 MPa foram restritivos para a cultura do trigo (Merotto \& Mundstock, 1999).

O objetivo deste trabalho foi avaliar a qualidade estrutural de um Latossolo Roxo sob os sistemas de plantio direto e cultivo convencional. 


\section{MATERIAL E MÉTODOS}

O estudo foi realizado na fazenda São Manuel, localizada no Município de Bela Vista do Paraíso, PR. Segundo Köppen, o clima da região é do tipo CWA. O solo é classificado como Latossolo Roxo distrófico A moderado, textura muito argilosa, fase floresta subtropical subperenifólia, relevo suave ondulado.

Os tratamentos constituíram-se de sistemas de manejo, a saber: soja sob plantio direto de longa duração (17 anos) com sucessão trigo/soja e safrinha de milho; soja sob cultivo convencional (10 anos), compreendendo uma aração e duas gradagens, sendo uma após a aração, e a outra, por ocasião do plantio; e como testemunha, o solo sob floresta nativa.

Nas profundidades de $0-20 \mathrm{~cm}$ (horizonte A) e $60-80 \mathrm{~cm}$ (horizonte $\mathrm{Bw}$ ) foram coletadas amostras para análises de caracterização. Foram quantificados os teores de gibbsita $(\mathrm{Gb})$ e caulinita $(\mathrm{Ct})$ na fração argila desferrificada pela análise térmica diferencial, e os de óxidos $\left(\mathrm{SiO}_{2}, \mathrm{Al}_{2} \mathrm{O}_{3}\right.$ e $\left.\mathrm{Fe}_{2} \mathrm{O}_{3}\right)$ através do ataque sulfúrico, na terra fina (Embrapa, 1997). A análise granulométrica foi realizada pelo método da pipeta (Day, 1965). Os resultados destas análises são apresentados na Tabela 1.

Nas profundidades de $0-20 \mathrm{~cm}$ e $20-40 \mathrm{~cm}$, determinou-se o pH e o teor de carbono orgânico (C org.), e calculou-se a matéria orgânica $(\mathrm{MO}=\mathrm{C}$ org. $\mathrm{x} 1,724)$, além da argila dispersa em água e conseqüente cálculo do índice de floculação (IF), segundo Embrapa (1997). Determinaram-se os teores de óxidos de ferro livres totais por ditionito-citrato-bicarbonato de sódio (DCB), conforme Mehra \& Jackson (1960), e as formas menos cristalinas, pelo oxalato de amônio ácido (OXA), segundo Schwertmann (1964). Determinaram-se também as formas de óxidos de ferro extraídas pelo pirofosfato de sódio (P) (Sheldrick \& McKeague, 1975) nos agregados, objetivando obter os teores complexados com a fração orgânica do solo. A determinação do ferro nos extratos foi feita por espectrofotometria de absorção atômica (Tabela 2).
Agregados com diâmetro entre 4,76 e 7,93 mm foram obtidos através de peneiramento de material indeformado, amostrado na camada superficial $(0-20 \mathrm{~cm})$ e subsuperficial $(20-40 \mathrm{~cm})$ do solo conforme Kemper \& Rosenau (1986). A estabilidade de agregados foi determinada através de peneiramento em água, após pré-umedecimento lento, por capilaridade. O diâmetro médio geométrico (DMG) foi calculado segundo Kemper \& Rosenau (1986).

No método para medir a resistência dos agregados ao impacto das gotas de chuva simulada, utilizaram-se agregados com diâmetro semelhante aos utilizados no teste de estabilidade (60 agregados/amostra), que foram colocados num suporte com abertura de $4 \mathrm{~mm}$ sob o gotejador com frequiência constante ( $91 \pm 5$ gotas por minuto), até que os agregados fossem destruídos. Os agregados foram considerados destruídos quando passaram pela abertura do suporte. Foi registrado o número de gotas necessárias para destruir os agregados. Os agregados não destruídos após 5 minutos de exposição foram classificados como resistentes. O método baseou-se em trabalho de Bruce-Okine \& Lal (1975), com modificações de Silva et al. (1995). Após o teste, calculou-se a energia cinética total necessária para a destruição dos agregados, multiplicando-se a energia cinética gerada por uma gota pelo número de gotas necessárias para destruir os agregados. O cálculo da energia cinética foi realizado segundo Silva et al. (1995), com modificações propostas por Silva et al. (1998). $\mathrm{O}$ resultado final foi expresso em $\mathrm{J} \mathrm{kg}^{-1}$ de agregado.

$\mathrm{O}$ teste de resistência à penetração (RP) foi determinado horizontalmente com micropenetrômetro com ponta de $6,4 \mathrm{~mm}$ de diâmetro (mod. Yamanaka, DIK5551), nas profundidades de 0-5, 5-20, 20-40 e 40-60 cm, na face da trincheira aberta na linha de plantio em cada unidade experimental, segundo Bradford (1986), e os valores em kg cm$~^{-2}$ foram multiplicados por 0,31 para transformação em MPa (Arshad et al., 1996). Na Tabela 3 são apresentadas as classes de resistência à penetração adaptadas de Soil Survey Staff (1993), citado por Arshad et al. (1996). A umidade do solo no momento dos testes estava próxima da capacidade de campo.

TABELA 1. Atributos mineralógicos, químicos e físicos do Latossolo Roxo de Bela Vista do Paraíso, PR.

\begin{tabular}{ccccccccccccc}
\hline Horizonte & $\begin{array}{c}\text { Prof. } \\
(\mathrm{cm})\end{array}$ & $\mathrm{Ct}$ & $\mathrm{Gb}$ & $\mathrm{SiO}_{2}$ & $\mathrm{Al}_{2} \mathrm{O}_{3}$ & $\mathrm{Fe}_{2} \mathrm{O}_{3}$ & $\mathrm{Ki}$ & $\mathrm{Kr}$ & $\mathrm{A}$ & $\begin{array}{c}\mathrm{S} \\
\mathrm{AF}\end{array}$ & $\mathrm{AG}$ \\
\hline $\mathrm{A}$ & $0-20$ & 280 & 150 & 191 & 214 & 262 & 1,52 & 0,85 & 500 & 320 & 80 & 100 \\
$\mathrm{Bw}$ & $60-80$ & 290 & 153 & 262 & 239 & 264 & 1,87 & 1,09 & 740 & 160 & 60 & 40 \\
\hline
\end{tabular}

${ }^{1} \mathrm{Ct}$ : caulinita; Gb: gibbsita; Ki: relação molecular $\mathrm{SiO}_{2} / \mathrm{Al}_{2} \mathrm{O}_{3}$; $\mathrm{Kr}$ : relação molecular $\mathrm{SiO}_{2} /\left(\mathrm{Al}_{2} \mathrm{O}_{3}+\mathrm{Fe}_{2} \mathrm{O}_{3}\right)$; A: argila; S: silte; $\mathrm{AF}$ : areia fina; $\mathrm{AG}$ : areia grossa. 
TABELA 2. Atributos químicos e índice de floculação do Latossolo Roxo de Bela Vista do Paraíso, PR, em duas profundidades ${ }^{1}$.

\begin{tabular}{|c|c|c|c|c|c|c|}
\hline \multirow[t]{3}{*}{ Sistema de cultivo } & \multirow[t]{3}{*}{$\mathrm{pH}$} & \multirow[t]{2}{*}{ MO } & \multicolumn{3}{|c|}{$\mathrm{Fe}_{2} \mathrm{O}_{3}$} & \multirow{3}{*}{$\begin{array}{c}\text { IF } \\
(\%)\end{array}$} \\
\hline & & & DCB & OXA & $P$ & \\
\hline & & ------- & - ( & & & \\
\hline & \multicolumn{6}{|c|}{$0-20 \mathrm{~cm}$} \\
\hline Plantio direto & $6,1 \mathrm{a}$ & $42,0 \mathrm{~b}$ & $129,4 \mathrm{a}$ & $11,0 \mathrm{a}$ & $0,66 \mathrm{~b}$ & $58,4 \mathrm{~b}$ \\
\hline Cultivo convencional & $5,4 \mathrm{c}$ & $22,6 \mathrm{c}$ & $127,4 \mathrm{~b}$ & $10,2 b$ & $0,38 \mathrm{c}$ & $45,6 \mathrm{c}$ \\
\hline \multirow[t]{2}{*}{ Floresta nativa } & $6,0 \mathrm{~b}$ & $47,0 \mathrm{a}$ & $130,0 \mathrm{a}$ & $11,0 \mathrm{a}$ & $1,98 \mathrm{a}$ & $59,6 \mathrm{a}$ \\
\hline & \multicolumn{6}{|c|}{$20-40 \mathrm{~cm}$} \\
\hline Plantio direto & $5,1 b$ & $5,6 \mathrm{a}$ & $111,4 b$ & $13,8 \mathrm{a}$ & $0,10 \mathrm{a}$ & $98,6 \mathrm{a}$ \\
\hline Cultivo convencional & $6,3 \mathrm{a}$ & $5,4 \mathrm{a}$ & $130,0 \mathrm{a}$ & $13,0 \mathrm{~b}$ & $0,10 \mathrm{a}$ & $96,0 \mathrm{~b}$ \\
\hline Floresta nativa & $5,1 b$ & $6,4 \mathrm{a}$ & $129,0 \mathrm{a}$ & $14,0 \mathrm{a}$ & $0,16 \mathrm{a}$ & $98,2 \mathrm{a}$ \\
\hline
\end{tabular}

${ }^{1}$ Médias seguidas pela mesma letra, na camada de 0-20 cm e na de 20-40 cm, não diferem entre si a 5\% de probabilidade pelo teste de Tukey; MO: matéria orgânica; DCB: ditionito-citrato-bicarbonato de sódio; OXA: oxalato de amônio ácido; P: pirofosfato de sódio; IF: índice de floculação.

TABELA 3. Classes de resistência à penetração'.

\begin{tabular}{lc}
\hline Classe & $\begin{array}{c}\text { Resistência à penetração } \\
(\mathrm{MPa})\end{array}$ \\
\hline Extremamente baixa & $<0,01$ \\
Muito baixa & $0,01-0,1$ \\
Baixa & $0,1-1,0$ \\
Moderada & $1,0-2,0$ \\
Alta & $2,0-4,0$ \\
Muito alta & $4,0-8,0$ \\
Extremamente alta & $>8,0$ \\
\hline
\end{tabular}

${ }^{1}$ Adaptado de Soil Survey Staff (1993), citado por Arshad et al. (1996).

O delineamento experimental foi inteiramente casualizado com parcelas subdivididas, com cinco repetições. As parcelas experimentais foram constituídas pelos sistemas de manejo, e as subparcelas, pelas profundidades de amostragens.

Os dados foram submetidos à análise de variância, e a comparação entre as médias dos sistemas de manejo e das profundidades foi feita pelos testes de Tukey e t, respectivamente, a $5 \%$ de probabilidade.

\section{RESULTADOS E DISCUSSÃO}

Na camada superficial, os maiores valores de índice de floculação foram observados na floresta nativa, seguidos pelos do plantio direto; estes valores estão relacionados com os maiores teores de matéria orgânica e formas de $\mathrm{Fe}$, notadamente o Fe ligado à fração orgânica, cujo extrator é o pirofosfato de sódio (Tabela 2). Os valores de índice de floculação da camada subsuperficial foram bastante elevados em relação aos da camada superficial, sendo, a diferença, significativa; e apresentaram comportamento semelhante aos da camada superficial em relação aos sistemas de manejo; entretanto, a explicação deste comportamento não pode ser atribuída à matéria orgânica, como observado na camada superficial, devido aos baixos valores observados, e sim a aspectos ligados à gênese e mineralogia do solo estudado.

A porcentagem de agregados menores e maiores que $2 \mathrm{~mm}$ foram constatadas, respectivamente, nas camadas superficial e subsuperficial (Fig. 1). Na camada superficial, as maiores porcentagens de agregados menores que 2,0 $\mathrm{mm}$ foram observadas no sistema de cultivo convencional; estes resultados estão coerentes com seus menores teores de matéria orgânica, do Fe ligado à fração orgânica, e ao índice de floculação (Tabela 2). Na camada subsuperficial, a maior porcentagem foi observada na floresta nativa, porém esta não apresentou diferenças significativas em relação a outros sistemas, e os atributos químicos aqui relacionados não foram suficientes para explicar esta tendência (Silva \& Mielniczuk, 1998). O plantio direto e a floresta nativa, na camada superficial, apresentaram as maiores porcentagens de agregados nas classes de maior diâmetro (2,0-7,9 mm), em torno de $80 \%$ (Fig. 1). Harris et al. (1996) utilizaram a porcentagem de agregados estáveis $(>2 \mathrm{~mm})$ 
em água para avaliar a qualidade do solo em sistemas de manejo; segundo esses autores os valores foram 40, 26 e 19\%, em sistema conservacionista, plantio direto e solo arado, respectivamente. Apenas no sistema convencional foram observadas diferenças significativas entre as profundidades estudadas com relação aos agregados menores e maiores que $2 \mathrm{~mm}$. Os resultados deste trabalho sugerem que o preparo intensivo a que o solo foi submetido no cultivo convencional, durante dez anos, contribuiu para a redução da estabilidade dos agregados, quando comparado com o plantio direto, onde o solo foi pouco revolvido (Machado et al., 1981; Reinert et al., 1984; Harris et al., 1996). Na profundidade de $20-40 \mathrm{~cm}$, não houve diferenças significativas entre os tratamentos.

Os valores do diâmetro médio geométrico apresentaram uma variação de 2,419 a $1,764 \mathrm{~mm}$ $(0-20 \mathrm{~cm})$ e 2,133 a 1,638 mm (20-40 cm) (Fig. 1). Os valores do diâmetro médio geométrico mostra- ram a mesma tendência de comportamento da classe de agregados maiores que $2 \mathrm{~mm}$, indicando, conforme Alvarenga et al. (1986) e Castro Filho et al. (1998), que esta classe pode ser utilizada para expressar a agregação do solo.

Na camada de 0-20 cm, o cultivo convencional apresentou os menores valores de diâmetro médio geométrico; esses resultados podem ser atribuídos aos seus teores mais reduzidos de matéria orgânica e ao preparo do solo (Tabela 2), corroborando observações de Carpenedo \& Mielniczuk (1990), Campos et al. (1995) e Da Ros et al. (1997). Por outro lado, na camada subsuperficial, o cultivo convencional apresentou o maior valor de diâmetro médio geométrico. Estes resultados se devem ao fato de que provavelmente no sistema de cultivo convencional, por ação de compressão das partículas do solo, e pela ação do arado e da grade, houve a formação de torrões na massa do solo, conforme observações no campo, sem, entretanto, ocorrerem os mecanismos que
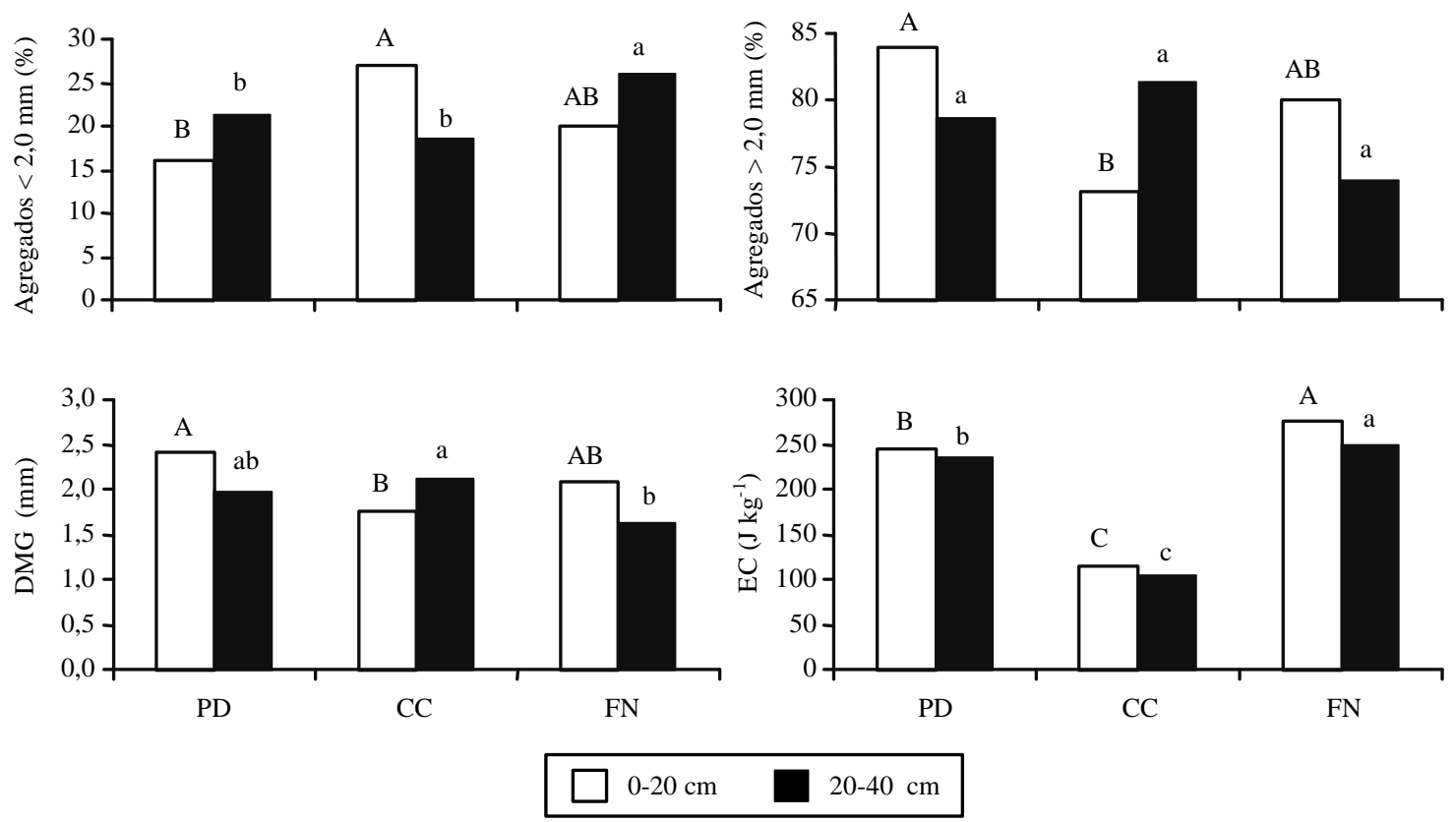

FIG. 1. Distribuição, diâmetro médio geométrico (DMG) dos agregados e energia cinética (EC) necessária para destruir os agregados, nos sistemas plantio direto (PD), cultivo convencional (CC) e floresta nativa (FN) em Latossolo Roxo. Médias com a mesma letra, maiúscula na camada superficial e minúscula na camada subsuperficial, não diferem entre si a $5 \%$ de probabilidade pelo teste de Tukey. 
contribuem para a estabilização desses torrões, não apresentando as qualidades positivas de um agregado (Silva \& Mielniczuk, 1998). Foram observadas diferenças significativas entre as profundidades estudadas para o diâmetro médio geométrico.

O método da energia cinética necessária para destruir os agregados proporcionou maior discriminação entre os sistemas estudados do que a estabilidade de agregados expressa pelo diâmetro médio geométrico (Fig. 1). Segundo Angulo et al. (1984) e Silva et al. (1998), o método da energia cinética parece reproduzir melhor as forças de campo responsáveis pela desintegração da estrutura do solo. Na camada superficial, os maiores valores de energia cinética foram observados na floresta nativa seguida do plantio direto, e as diferenças foram significativas; estes resultados estão de acordo com os teores de matéria orgânica, Fe ligado à fração orgânica, e índice de floculação (Tabela 2). Na camada subsuperficial, os resultados de energia cinética apresentaram as mesmas tendências; entretanto, os atributos químicos e físicos abordados neste estudo (Tabela 2) foram insuficientes para explicar estas variações (Silva \& Mielniczuk, 1998). Apenas no sistema convencional não se observaram diferenças significativas entre as profundidades estudadas.

Na camada superficial, o diâmetro médio geométrico e a energia cinética no sistema plantio direto foram cerca de 1,37 e 2,4 vezes maiores, respectivamente, do que no cultivo convencional; o plantio direto propiciou agregados maiores e mais resistentes (Fig. 1). Os resultados relativos ao diâmetro médio geométrico e energia cinética no sistema floresta nativa estão de acordo com a mineralogia do solo estudado (Tabela 1), onde o predomínio de caulinita está associado a menores valores desses parâmetros em comparação à gibbsita, uma vez que Silva et al. (1998), trabalhando com Latossolos gibbsíticos, encontraram valores de energia cinética e diâmetro médio geométrico, em cerrado nativo, superiores a $300 \mathrm{~J} \mathrm{~kg}^{-1}$ e 4,0 mm, respectivamente.

Os valores de resistência à penetração refletiram a influência dos sistemas de manejo no arranjo das unidades estruturais, ao longo do perfil (Fig. 2). Houve variação de 0,15 a 2,91 MPa; segundo Arshad et al. (1996), estes valores estão compreendidos entre as classes baixa e alta (Tabela 3). Os maiores valores de resistência à penetração foram constatados no cultivo convencional, sendo críticos na camada de 5 a $20 \mathrm{~cm}$, com valores superiores a $2 \mathrm{MPa}$ no cultivo convencional e plantio direto (Fig. 2). Segundo Grant \& Lanfond (1993), Arshad et al. (1996) e Merotto \& Mundstock (1999), valores superiores a 2 MPa indicam presença de camadas compactadas, atuando como uma barreira para o desenvolvimento radicular, sendo que este efeito é resultante do preparo do solo com uso de grade pesada ao longo dos anos. No plantio direto, apesar de não se ter revolvimento do solo como no cultivo convencional, há um tráfego de máquinas que possivelmente esteja ocasionando a compactação. A presença de camadas compactadas em sistemas de plantio direto e convencional na faixa de 5-20 cm foi observada por Vieira \& Muzilli (1984) e Corrêa (1985). Os valores elevados de resistência à penetração na camada de 5-20 cm no plantio direto não chegam a comprometer o sistema, devido à maior continuidade de poros, homogeneidade do solo e à maior atividade microbiana, ponderando-se valores superiores em plantio direto (Grant \&

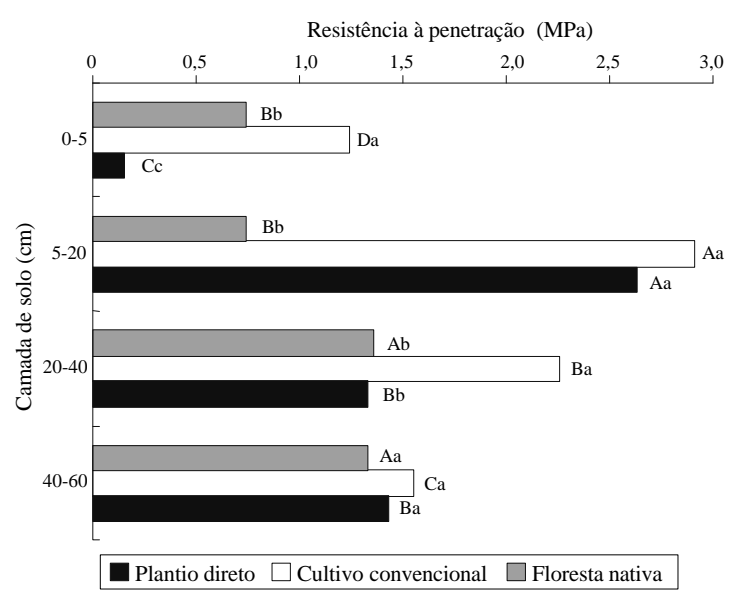

FIG. 2. Resistência à penetração em diferentes camadas do Latossolo Roxo, nos sistemas plantio direto, cultivo convencional e floresta nativa. Médias com a mesma letra, maiúscula (comparação entre sistemas de manejo) e minúscula (comparação entre sistemas de manejo dentro de cada profundidade), não diferem entre si a 5\% de probabilidade pelo teste de Tukey. 
Lanfond, 1993; Arshad et al., 1996). Por outro lado, na camada de $0-5 \mathrm{~cm}$, o plantio direto apresentou valor baixo de resistência à penetração, o que está relacionado com seu teor elevado de matéria orgânica (Tabela 2) e com material vegetal em decomposição na superfície do solo. A floresta nativa apresentou valores de resistência à penetração mais uniformes em todo o perfil; segundo Arshad et al. (1996) esses valores foram enquadrados nas classes baixa e moderada. Na camada de 40-60 cm as diferenças entre os tratamentos não foram significativas, e os valores foram enquadrados na classe moderada (Arshad et al., 1996).

\section{CONCLUSÕES}

1. O sistema plantio direto propiciou agregados maiores e mais resistentes na camada superficial.

2. Valores críticos de resistência à penetração foram observados no cultivo convencional e plantio direto na camada de $5-20 \mathrm{~cm}$.

3. Os atributos químicos e físicos do solo apresentaram bom desempenho na avaliação da qualidade estrutural do Latossolo Roxo no tocante aos sistemas de manejo estudados.

\section{REFERÊNCIAS}

ALVARENGA, R.C.; FERNANDES, B.; SILVA, T.C.A.; RESENDE, M. Estabilidade de agregados de um Latossolo Roxo sob diferentes métodos de preparo do solo e de manejo da palhada do milho. Revista Brasileira de Ciência do Solo, Campinas, v.10, p.273-277, 1986.

ANGULO, R.J.; ROLOFF, G.; SOUZA, M.L.P. Correlação entre diferentes formas de determinação e representação da estabilidade e resistência dos agregados do solo. Revista Brasileira de Ciência do Solo, Campinas, v.8, p.7-12, 1984.

ARSHAD, M.A.; LOWERY, B.; GROSSMAN, B. Physical tests for monitoring soil quality. In: DORAN, J.W.; JONES, A.J. (Ed.). Methods for assessing soil quality. Madison : Soil Science Society of America, 1996. p.123-141. (SSSA Special Publication, 49).

BRADFORD, J.M. Penetrability. In: KLUTE, A. (Ed.). Methods of soil analysis. 2.ed. Madison : American Society of Agronomy, 1986. v.1, p.443-460.
BRUCE-OKINE, E.; LAL, R. Soil erodibility as determined by raindrop technique. Soil Science, Baltimore, v.119, n.2, p.149-157, 1975.

CAMPOS, B.C.; REINERT, D.J.; NICOLODI, R.; RUEDELL, J.; PETRERE, C. Estabilidade estrutural de um Latossolo Vermelho-Escuro distrófico após sete anos de rotação de culturas e sistemas de manejo de solo. Revista Brasileira de Ciência do Solo, Campinas, v.19, n.1, p.121-126, jan./abr. 1995.

CARPENEDO, V.; MIELNICZUK, J. Estado de agregação e qualidade de agregados de Latossolos Roxos, submetidos a diferentes sistemas de manejo. Revista Brasileira de Ciência do Solo, Campinas, v.14, n.1, p.99-105, jan./abr. 1990.

CASTRO FILHO, C.; MUZILLI, O.; PODANOSCHI, A.L. Estabilidade dos agregados e sua relação com o teor de carbono orgânico num Latossolo Roxo distrófico, em função de sistemas de plantio, rotações de culturas e métodos de preparo das amostras. Revista Brasileira de Ciência do Solo, Viçosa, v.22, n.3, p.527-538, jul./set. 1998.

CORRÊA, J.C. Características físicas de um Latossolo Amarelo muito argiloso (Typic Acrorthox) do Estado do Amazonas, sob diferentes métodos de preparo do solo. Pesquisa Agropecuária Brasileira, Brasília, v.20, n.12, p.1381-1387, dez. 1985.

DA ROS, C.O.; SECCO, D.; FIORIN, J.E.; PETRERE, C.; CADORE, M.A.; PASA, L. Manejo do solo a partir de campo nativo: efeito sobre a forma e estabilidade da estrutura ao final de cinco anos. Revista Brasileira de Ciência do Solo, Campinas, v.21, n.2, p.241-247, abr./jun. 1997.

DAY, P.R. Particle fractionation and particle-size analysis. In: BLACK, C.A. (Ed.). Methods of soil analysis. Madison: American Society of Agronomy, 1965. v.1, p.545-566.

EMBRAPA. Centro Nacional de Pesquisa de Solos (Rio de Janeiro, RJ). Manual de métodos de análise de solo. 2.ed.rev.atual. Rio de Janeiro, 1997. 212p.

GRANT, C.A.; LANFOND, G.O. The effects of tillage systems and crop sequences on soil bulk density and penetration resistance on a clay soil in Southern Saskatchewan. Canadian Journal of Soil Science, Ottawa, v.73, n.2, p.223-232, May 1993.

HARRIS, R.F.; KARLEN, D.L.; MULLA, D.J. A conceptual framework for assessment and management of soil quality and health. In: DORAN, J.W.; JONES, A.J. (Ed.). Methods for assessing soil 
quality. Madison : Soil Science Society of America, 1996. p.61-82. (SSSA Special Publication, 49).

KEMPER, W.D.; ROSENAU, R.C. Aggregate stability and size distribution. In: KLUTE, A. (Ed.). Methods of soil analysis. Madison : American Society of Agronomy, 1986. part 1, p.425-442. (ASA Agronomy, 9).

MACHADO, J.A.; SOUZA, D.M.P.; BRUM, A.C.R. Efeito de anos de cultivo convencional em propriedades físicas do solo. Revista Brasileira de Ciência do Solo, Campinas, v.5, n.3, p.181-189, set./dez. 1981.

MEHRA, O.P.; JACKSON, M.L. Iron oxide removal from soils and clays by a dithionite-citrate system buffered with sodium bicarbonate. Clays and Clay Minerals, Boulder, v.3, p.317-327, 1960.

MEROTTO JUNIOR, A.; MUNDSTOCK, C.M. Wheat root growth as affected by soil strength. Revista Brasileira de Ciência do Solo, Viçosa, v.23, n.2, p.197-202, abr./jun. 1999.

REINERT, D.J.; MUTTI, L.S.M.; ZAGO, A.; AZOLIN, M.A.D.; HOFFMANN, C.L. Efeito de diferentes métodos de preparo do solo sobre a estabilidade de agregados em solo Podzólico Vermelho-Amarelo. Revista do Centro de Ciências Rurais, Santa Maria, v.14, p.19-25, 1984.

SCHWERTMANN, U. Differenzierung der Eisenoxide des Bodens durch Extraktion mit Ammoniumoxalat-
Lösung. Zeitschrift für Pflanzenernährung, Düngung Bodenkunde, Weiheim, v.105, n.3, p.194202, 1964.

SHELDRICK, B.H.; McKEAGUE, J.A. A comparison of extractable $\mathrm{Fe}$ and $\mathrm{Al}$ data using methods followed in the USA and Canada. Canadian Journal of Soil Science, Ottawa, v.55, n.1, p.77-78, Feb. 1975.

SILVA, I.F.; MIELNICZUK, J. Sistemas de cultivo e características do solo afetando a estabilidade de agregados. Revista Brasileira de Ciência do Solo, Viçosa, v.22, n.2, p.311-317, abr./jun. 1998.

SILVA, M.L.N.; BLANCANEAUX, P.; CURI, N.; LIMA, J.M.; MARQUES, J.J.G.S.M.; CARVALHO, A.M. Estabilidade e resistência de agregados de Latossolo Vermelho-Escuro cultivado com sucessão milhoadubo verde. Pesquisa Agropecuária Brasileira, Brasília, v.33, n.1, p.97-103, jan. 1998.

SILVA, M.L.N; CURI, N.; MARQUES, J.J.G.S.M.; LIMA, L.A.; FERREIRA, M.M.; LIMA, J.M. Resistência ao salpico provocado por impacto de gotas de chuva simulada em Latossolos e sua relação com características químicas e mineralógicas. Ciência e Prática, Lavras, v.19, n.2, p.182-188, 1995.

VIEIRA, M.J.; MUZILLI, O. Características físicas de um Latossolo Vermelho-Escuro sob diferentes sistemas de manejo. Pesquisa Agropecuária Brasileira, Brasília, v.19, n.7, p.873-882, jul. 1984. 